\title{
ROCK PHYSICS INVESTIGATION OF SEISMIC WAVE ABSORPTION IN RESERVOIR ROCKS
}

\author{
W. O. RAJI AND A. RIETBROCK \\ (Received 2, January 2009; Revision Accepted 19, January 2009)
}

\begin{abstract}
This research provides insight into the process of pore- fluid induced absorption which is important for absorption compensation and seismic image enhancement. The absorptive property of a medium is described by the rock inverse quality factor $\left(Q^{-1}\right)$, perturbational forward modeling is conducted by varying saturation and estimating the inverse quality factor using first principles of rock inelastic properties and modeling seismic absorption in rock with partial gas saturation as commonly encountered in hydrocarbon exploration. The response of a visco-elastic rock depends on the frequency of the propagating seismic energy. The difference between modulus estimated at high and low frequencies is translated to the coefficient of absorption (or inverse quality factor). Absorption estimated at 0.4 irreducible water saturation ( 0.6 gas saturation) is higher than 0.8 irreducible water saturation ( 0.2 gas saturation). The higher the gas saturation, the higher the absorption, and the poorer the seismic image. The signature of gas induced absorption in the selected earth model are the increase time -thickness in the reservoir interval, large contrast in the top and base amplitude, pulse broadening and wavelet distortion. In hydrocarbon reservoir evaluation, seismic absorption can be used to interpret for fluid units. Information about seismic absorption can also be used, by means of absorption compensation, to enhance seismic data resolution.
\end{abstract}

KEYWORDS: Saturation, Absorption, Dispersion, Inverse Quality Factor, Anelastic,

\section{INTRODUCTION}

Seismic absorption has become a field of extensive research in the oil and gas industry in recent years due to the requirement of obtaining seismic images with high resolution and accuracy from great depth and areas of complex geology. Seismic signals acquired over hydrocarbon reservoirs usually exhibit abnormal amplitude reduction compared to the ones acquired from the surrounding rocks. This unusual amplitude reduction is attributed to the absorptive properties of the fluid filling the pore spaces of the rock. Mathematical model arising from modifications to the elastic wave equations, like the standard solid linear models, Futterman model, Lomnitz model have been used to explain the phenomenon of seismic wave absorption in a dissipative media. More complicated models like Dvorkin et al, 2003; Pride et al, 2004 relate seismic absorption to the porous properties of the rock materials, such as porosity, permeability, saturation and fluid content.

Absorption is a phenomenon that is responsible for the reduction in the amplitude of a seismic energy as it propagates through the earth. Based on mechanism, seismic absorption has been classified in to two: Extrinsic absorption and intrinsic absorption. Extrinsic absorption is the redistribution of seismic wave energy within the propagation medium but does not necessary remove energy from the overall wave field. The energy is only redistributed among the reflected and transmitted secondary waves. In intrinsic absorption (which is the focus of this research), part of the energy of the wave is irreversibly converted into heat during the oscillatory fluid flow and sliding (frictional) movement induced by the propagating seismic waves.

Perturbational forward modeling is conducted by varying the reservoir properties such as saturation and fluid content to estimate the corresponding absorption a seismic wave will experience while propagating in such reservoir. The underlying assumption is that if the seismic response is similar, the rock properties and conditions in the subsurface giving rise to this response is also similar. Basic rock anelastic principles (Gassmann, 1951; Mavko et al, 1995; 1998, Dvorkin and Mavko, 2006) were employed to study attenuation in heterogeneous clastic rock, estimate changes in dynamic moduli with frequency and measured the inverse quality factor using the standard solid linear model.

\section{MODULUS - FREQUENCY DISPERSION AND ATTENUATION AT PARTIAL SATURATION}

In partially saturated rock, viscoelastic effect and attenuation may arise from the oscillatory liquid cross flow between the fully saturated patches and the surrounding rock with partial gas saturation. The reaction of a rock with patchy saturation to loading due to elastic wave propagation depends on the frequency of the wave. If the frequency is low, loading will be slow, the oscillatory pore pressure between the fully liquid saturated patches and the domain next to it will reach equilibrium, the patch is "relaxed".

W. O. Raji, Department of Earth and Ocean Sciences, University of Liverpool, L69 3gp. Liverpool, United Kingdom

A. Rietbrock, Department of Earth and Ocean Sciences, University of Liverpool, L69 3gp. Liverpool, United Kingdom 


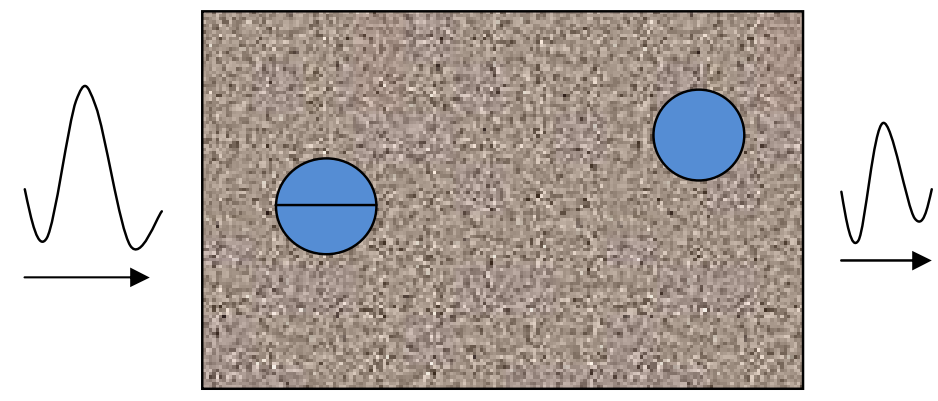

\section{Liquid $\left(\mathrm{H}_{2} \mathrm{O}\right)$ saturated patch $\square$ Rock with partial gas saturation}

Fig.1: Schematic diagram of microscopic fully saturated patches in partially saturated reservoir

If the frequency is high, loading will be high, the oscillatory pressure between the fully liquid -saturated patch and the surrounding rock will not attain equilibrium .The patch is "unrelaxed" (Dvorkin and Mavko, 2006). The elastic modulus of a partially saturated rock due to attenuation changes with frequency. The difference between high and low frequencies compressional moduli is translated into inverse quality factor using Standard Solid Linear Model of Dvorkin et al. (2003).

The compressional moduli of a partially saturated rock containing fully liquid saturated patches is estimated from the bulk moduli of a partially saturated rock $\left(k_{g a t 0}\right)$ and the shear modulus of the dry frame $\left(G_{D r y}\right)$ using Gasmann, (1951) model.

At low frequency, the compressional $\left(M_{\text {sato }}\right)$ modulus is estimated as

$M_{\operatorname{rat} 0}=k_{\operatorname{rat} 0}+\left(\frac{4}{3}\right) G_{D r y}$

Where $k_{\text {sato }}=k_{g} \frac{\phi k_{D r y}=(1+\phi) k_{f} k_{D r y} / k_{g}+k_{f}}{(1+\phi) k_{f}+\phi k_{g}-k_{f} k_{D r y} / k_{s}}$

$K_{D r y}$ and $K_{s} \quad$ are the shear modulus of dry frame and

mineral phase respectively, and $\boldsymbol{\phi}$ is the porosity. $K_{f}$ is

the bulk modulus of the mixed(water+ gas)fluid.

The effective bulk modulus of the pore fluid mix $\left(k_{f}\right)$ is the harmonic average of the modulus of water $\left(k_{w}\right)$ having volumetric concentration, sw and modulus of the gas $\left(k_{G}\right)$ with volumetric concentration, 1-sw.

$\frac{1}{k_{f}}=\frac{s w}{k_{W}}+\frac{1-s w}{k_{G}}$

At high frequency, the compressional modulus is calculated from the bulk and shear moduli.

$\frac{1}{k_{y s}+\left(\frac{4}{g}\right) G_{D r y}}=\frac{1}{k_{p}+\left(\frac{4}{g}\right) G_{D r y}}+\frac{1}{k_{g w=0}+\left(\frac{4}{g}\right) G_{D r y}}$
Where the bulk modulus of the fully saturated $\operatorname{patch}\left(k_{p}\right)$ and dry patch $\left(k_{s w=0}\right)$ are calculated as

$k_{\mathrm{p}}=k_{g} \frac{\phi k_{D r y}-(1+\phi) k_{w} k_{D r y} / k_{g}+k_{w}}{(1+\phi) k_{w}+\phi k_{g}-k_{w} k_{D r y} / k_{g}}$

(9)

$k_{s W=\emptyset}=k_{g} \frac{\phi k_{D r y}-(1+\phi) k_{G} k_{D r y} / k_{g}+k_{G}}{(1+\phi) k_{G}+\phi k_{g}-k_{G} k_{D r y} / k_{g}}$

The compressional modulus of saturated rock is, on the other hand, estimated from the dry frame $\left(M_{D r y}\right)$ using $V_{F}$-fluid substitution equation of Mavko et al. (1995; 1998)

At low frequency,

$M_{s a t 0}=M_{s} \frac{\phi M_{D r y}-(1+\phi) k_{f} M_{D r y} / M_{g}+k_{f}}{(1+\phi) k_{f}+\phi M_{g}-k_{f} M_{D r y} / M_{z}}$

At high frequency, the effective compressional modulus of the rock is estimated as the harmonic average of the compressional modulus of fully saturated $\left(M_{p}\right)$ patches and $\operatorname{dry}\left(M_{s w=0}\right)$ patches.

$\frac{1}{M_{\text {Iat }}}=\frac{s w}{M_{P}}+\frac{1-s w}{M_{\text {sw }}=0}$

Where

$$
\begin{gathered}
M_{p}=M_{g} \frac{\phi M_{D r y}-(1+\phi) k_{\mathrm{W}} M_{D r y} / M_{g}+k_{\mathrm{W}}}{(1+\phi) k_{\mathrm{W}}+\phi M_{z}-k_{\mathrm{W}} M_{D r y} / M_{z}} \\
M_{S w=0}=M_{g} \frac{\phi M_{D r y}-(1+\phi) k_{G} M_{D r y} / M_{g}+k_{G}}{(1+\phi) k_{G}+\phi M_{g}=k_{G} M_{D r y} / M_{g}}
\end{gathered}
$$

$M_{\text {dry }}$ and $M_{z}$ are the respective compressional modulus of the dry frame and the mineral phase.

The maximum inverse quality factor $\left(Q_{\max }^{-1}\right)$ is estimated from the compressional moduli at high and low frequencies as 
$Q^{-1}=\frac{M_{\text {Sat }}-M_{\text {sato }}}{2 \sqrt{M_{\text {Sato }} M_{\text {Sato }}}}$

$M_{\text {sat }}$ and $M_{\text {sato }}$ are the respective compressional modulus of the rock at high and low frequencies

\section{IRREDUCIBLE WATER SATURATION, $\boldsymbol{S}_{W r}$}

Real hydrocarbon reservoirs usually contain some quantities of water no matter the level of gas and / or oil charge. This water is referred to as irreducible water saturation, $s w_{r}$.

Given the volumetric concentration of the patch, $f_{p}$ and the bulk modulus of the pore fluid as

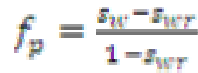

$\frac{1}{k_{f y}}=\frac{s w_{Y}}{k_{i q}}+\frac{1-s w_{Y}}{k_{G}}$ whens $s_{W} \leq s_{w r}$, the compressional modulus is estimated from (8), at $s_{w r}>s_{w r}$, the modulus is estimated (Gassmann, 1951) as,

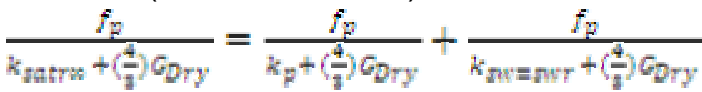

(15)

or from Vp-only fluid substitution, when $s_{W}>s_{W r}$ as

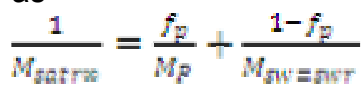

(16)

and from (12) whens $s_{w} s_{w r}$.

Given the parameters $M_{s w=s w r}$ and $M_{s w}=_{s w}$ as

$$
\begin{aligned}
& M_{s w=g w r}=M_{g} \frac{\phi M_{D r y}-(1+\phi) k_{f y} M_{D r y} / M_{t}+k_{f r}}{(1+\phi) k_{f r}+\phi M_{z}-k_{f r} M_{D r y} / M_{g}} \\
& k_{w}=s w r \\
& =k_{g} \frac{\phi k_{D r y}-(1+\phi) k_{f r} k_{D r y} / k_{g}+k_{f r}}{(1+\phi) k_{f r}+\phi k_{y}-k_{f} k_{D r y} / k_{y}}
\end{aligned}
$$
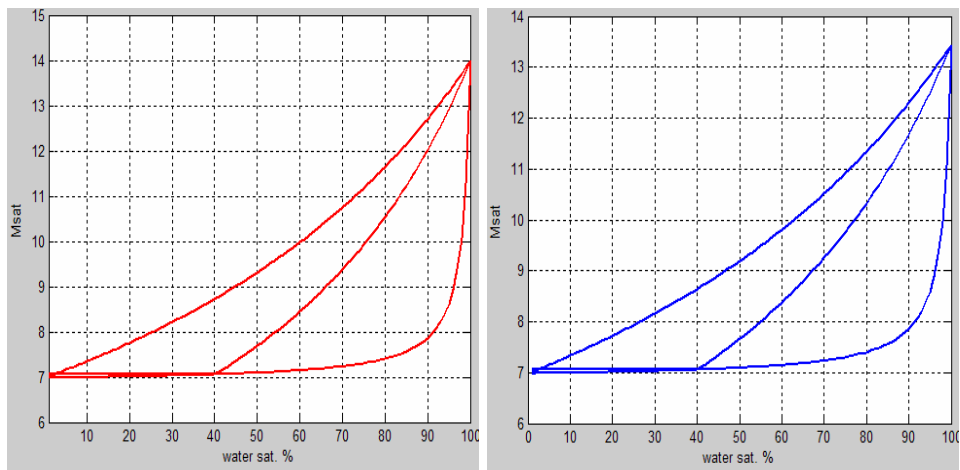

Fig.2: Compressional modulus at low and high frequencies versus water saturation with irreducible water saturation of 0.4 (middle line). Left (Gassman equation), right (Vp-only fluid substitution). The curve line is low frequency modulus while the nearly straight line is high frequency modulus.
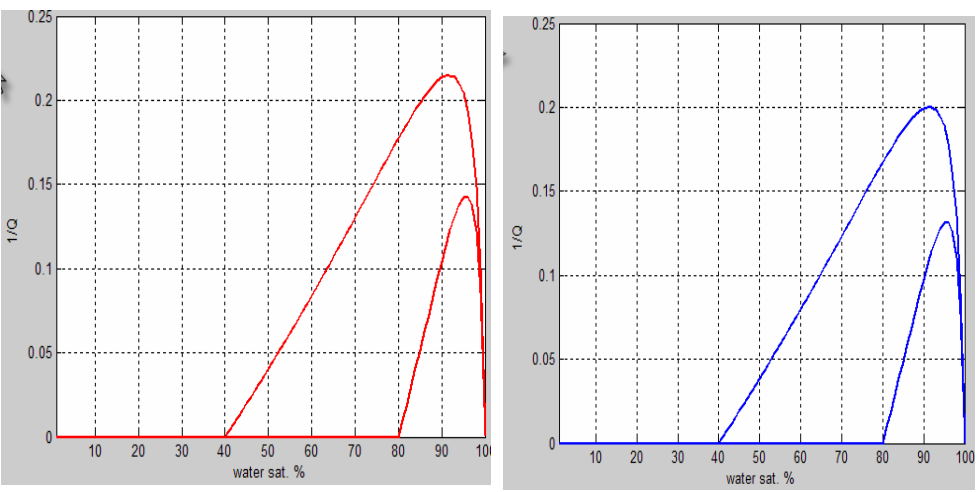

Fig.3: The maximum inverse quality factor versus irreducible water saturation (Swir) at Swir= 0.4 and 0.8, using Vp -only substitution (left) and Gassmann's equation (right) . 
An important conclusion about the effect of irreducible water saturation on absorption is that the higher the irreducible water saturation, the lower the absorption $Q^{-1}$ (Fig.3).

\section{SEISMIC WAVE ATTENUATION}

Rock property is described by a quality factor, $Q$. Both $Q$ and the velocity are used to describe the propagation of seismic energy in a rock. To demonstrate the effect of pore-fluid induced absorption in reservoir rocks, the spectrum of a seismic wavelet is modeled by the spectrum of a ricker wavelet and synthetic seismograms are computed in elastic earth model, representing rock with no pore fluid (Fig.4b) using the reflectivity method (Muller, 1985). To represent the effect of absorption due to pore fluid, seismograms from absorptive earth layer are computed by incorporating the Futteman (1962) causal absorption model into the reflectivity method. To model seismic absorption in rock, Different $Q^{-1}$ values were assigned to the reservoir layer and the layer's velocity is substituted with a complex frequency -dependent velocity.
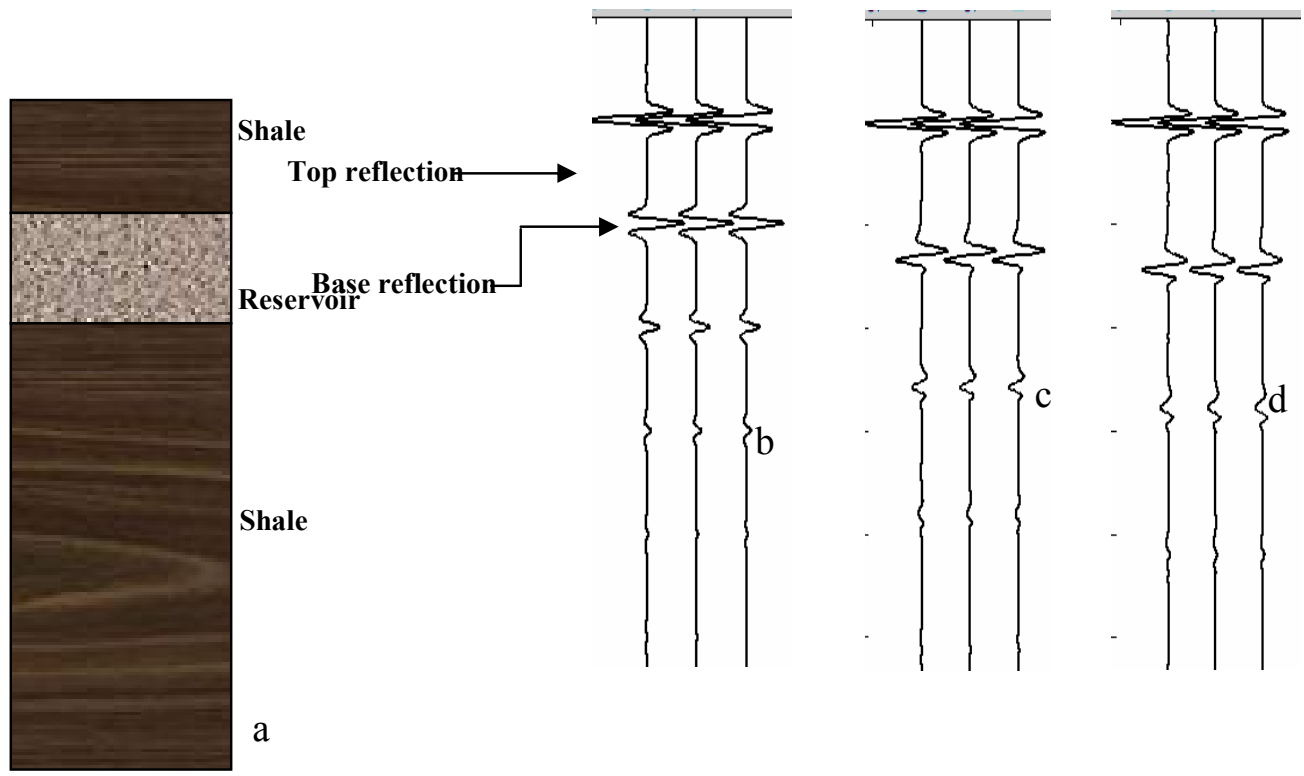

Fig.4: (a). Schematic diagram of a- three layered earth model (bottom to top: shale-clastic reservoir rock-shale) (b).P wave reflection for elastic model (dry rock,),(c) absorptive model using $Q$ value estimated at $0.4 S_{W R}$ saturation ,(d) absorptive model using $Q$ value estimated at $0.8 S_{W R}$

$\mathrm{P}-\mathrm{P}$ reflection is computed at the top and base of a reservoir in three -layer earth model elastic and anelastic/absorptive models consisting of shalesandstone-shale sequence. The two shale layers shared the same elastic property. For the elastic model, very large $(Q)$ quality factor (infinite absorption) is assigned to the shale layers while the various inverse maximum quality factor $\left(1 / Q_{\max }\right)$ estimated from the rock physics (Fig.3 left) were used to describe absorption in the reservoir rock using the Futterman (1962) causal absorption model,

$$
\begin{aligned}
& V_{(f)}=v_{(f)}-i \sigma_{(f)} / 2 \pi f \\
& v_{(f)}=v\left[1-\left(\pi Q_{0}\right)^{-1} \ln \left(f / f_{c}\right)\right] \\
& \sigma_{(f)}=\pi f / v Q_{0}
\end{aligned}
$$

The model link absorption to velocity dispersion and the velocity dispersion is frequency dependent. The computations for both elastic and absorptive models were effected in two separate MATLAB codes specially written for this study. The apparent increased time thickness in the reservoir interval, pulse broadening and wavelet distortion in addition to amplitude attenuation are the signatures of gas in the selected absorption model (Raji and Riebrock, 2009).

\section{DISCUSSION}

The amplitude spectrum of a seismic wavelet is modeled by the spectrum of a ricker wavelet and analytical relationship is derived to represent the effect of pore-fluid on the amplitude of reflected signal, using causal absorption formulation. For a particular pore fluid, the response of a visco-elastic rock to a propagating wave depends on the frequency of the wave, the difference between high and low frequency compressional moduli is translated to the inverse quality factor. The $Q$ values estimated from both Gassmann's and $\mathrm{Vp}$-fliud substitution at the same irreducible water saturation are comparable as shown in figure 3 and produce similar effect on the amplitude of the reflected waves in the reservoir interval. $\mathrm{P}$-wave absorption at 0.4 irreducible water saturation ( 0.6 gas saturation) is higher compared to $\mathrm{P}$-wave absorption at 0.8 irreducible water saturation ( 0.2 gas saturation). Amplitude absorption is higher at base of the reservoir compare to the top due to the presence of fluid. The contrast between top and bottom reflection (Figs. $4 \mathrm{c}$ and $\mathrm{d}$ ) is the absorptive effect of pore fluid on the propagating waves. 
CONCLUSION

Fluid in the pore spaces of reservoir rocks is responsible for the abnormal reduction in the amplitude of seismic wave returning from the base of reservoir rocks. The reduction in amplitude contributes to the poor seismic images resolution observed in seismic data gathered from reservoir rock during hydrocarbon exploration. Absorption depends on rock property and conditions as well as the frequency of the propagating seismic energy. In a reservoir with volumetric saturation of gas and irreducible water $\left(S_{w}+S_{G}=1\right)$ equal one, and where the water saturation equals the irreducible water saturation, the lower the water saturation, the higher the gas saturation, and the higher the absorption. Since seismic absorption produces poor seismic, information about seismic absorption can be used to enhance seismic resolution by means of absorption compensation. The apparent increased time -thickness in the reservoir interval, pulse broadening and wavelet distortion in addition to amplitude attenuation are the signatures of gas in the selected model.

\section{ACKNOWLEDGMENT}

This work is one of the products of a Ph.D research sponsored by petroleum Technology Development Fund, Nigeria. We thank PTDF. We are also grateful to the reviewers for their useful comments.

\section{REFERNCES}

Dvorkin, J. and G. Mavko, 2006. Modeling attenuation in reservoir and non-reservoir rocks. The Leading Edge. 25, pp.194-197.

Dvorking, J., Mavko, G., walls, J,, Taner, M.T. and Derzhi, N., 2003. Attenuation at patchy saturation- $A$ model. Proceeding of annual EAGE Convention. Stanvanger Norway, June 25.pp.Z-99.

Futterman, W. I., 1962. Dispersive body waves: Journal of Geophysics Research. 67, pp.5279-5291.

Gassmann, F., 1951. Uber die elastizitat poroser medien: Vierteljahrsschrift der Natur. Gesselschaft. 96, pp.1-23.

Mavko, G., Chan, C., and Mukerji, T., 1995. Fluid substitution: Estimating changes in $\mathrm{Vp}$ without knowing Vs. Geophysics. 60, pp.1750-1755

Mavko, G., Mukerji, T. and Dvorkin, J., 1998. Rock Physics Handbook, Cambridge University Press.pp.244-249.

Muller, G., 1985. The reflectivity method: A tutorial. Geophysics. 58, pp.153-174.

Pride, S. R., Berryman, J. G. and J. M. Harris, 2004. Seismic attenuation due to wave induced flow. Journal of Geophysical Research. 109, No.B1, B01201.

Raji, W.O., and Rietbrock, A., 2009. P-wave and S-wave seismic attenuation. British Geophysical Association-Research in Progress. Expanded abstract, pp.28. 